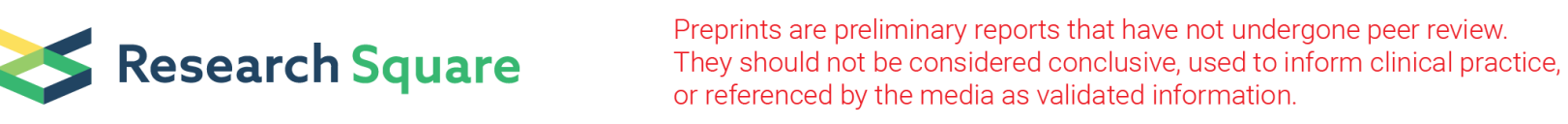

\title{
MicroRNA-935 mediates the regulatory effect of IncRNA KCNQ10T1 on tumor progression of breast cancer
}

\section{Chunjie Zhang}

Zibo Central Hospital

\section{Cuixue Gong}

Zibo Central Hospital

Jianzhao Li

Zibo Central Hospital

Jiaying Tang ( $\nabla$ novelty_cl@163.com )

Zibo Central Hospital

\section{Research}

Keywords: Breast cancer, KCNQ10T1, miR-935, Proliferation, Migration, Invasion, Diagnosis

Posted Date: April 8th, 2020

DOI: https://doi.org/10.21203/rs.3.rs-21089/v1

License: (c) (i) This work is licensed under a Creative Commons Attribution 4.0 International License. Read Full License 


\section{Abstract}

Background The biological function of long non-coding RNAs (IncRNAs) and microRNAs (miRNAs) have received increasing attention in the pathogenesis of various malignancies. This study aimed to evaluate the functional role and clinical significance of the KCNQ10T1/miR-935 axis in breast cancer (BCa) progression.

Methods Expression of KCNQ10T1 and miR-935 was estimated using quantitative real-time PCR (qRT$\mathrm{PCR}$ ). The interaction between KCNQ10T1 and miR-935 was confirmed by luciferase reporter assay. BCa cell proliferation, migration and invasion were evaluated using CCK-8 and Transwell assays. The clinical significance of the KCNQ10T1/miR-935 axis in BCa diagnosis was examined by ROC analysis.

Results The expression of KCNQ10T1 was significantly elevated, but the miR-935 expression was decreased in BCa cells (all P < 0.01). KCNQ10T1 could directly bind to miR-935, leading to the decreased miR-935 expression in $\mathrm{BCa}$ cells $(\mathrm{P}<0.001)$. The overexpression of miR-935 could inhibit BCa cell proliferation, migration and invasion, and remarkably reversed the regulatory effect of KCNQ1OT1 on BCa cell biological processes (all $\mathrm{P}<0.05$ ). Serum KCNQ10T1 levels were negatively correlated with miR-935 levels in BCa patients. The aberrant expression of KCNQ10T1 and miR-935 had relatively high diagnostic accuracy for the screening of BCa patients.

Conclusion In conclusion, miR-935 expression is downregulated in $\mathrm{BCa}$ cells, which can be inhibited by KCNQ10T1 and mediates the promoting effect of KCNQ10T1 on BCa tumor progression in vivo. Serum reduced expression of miR-935 may serve as a diagnostic biomarker of $\mathrm{BCa}$, and the KCNQ10T1/miR935 axis provides potential therapeutic targets for $\mathrm{BCa}$ treatment.

\section{Introduction}

Breast cancer $(\mathrm{BCa})$ is a common malignancy in female, which is a serious threat to women's life and health (1). According to statistic, breast cancer-associated mortality is the second highest among cancerassociated mortality around the world (2). Currently, some advanced tumor therapeutic strategies and technologies have been conducted, and the incidence of $\mathrm{BCa}$ is less than before (3). However, the survival outcomes of patient with $\mathrm{BCa}$ remain dismal. The understanding of the pathologic mechanisms in $\mathrm{BCa}$ development and progression is far from enough, which limits the development of efficient therapeutic approaches for $\mathrm{BCa}$ (4). Therefore, identifying key molecules that involving in the pathogenesis of $\mathrm{BCa}$ may provide new ideas to improve disease therapy.

In recent decades, numerous non-coding RNAs have received increasing attention on their important regulatory roles in various pathological processes, especially in human cancers $(5,6)$. Long non-coding RNAs (IncRNAs) and microRNAs (miRNAs) are considered two groups important non-coding RNAs. LncRNAs are a kind of transcript with more than 200 nucleotides in length, and miRNAs are a series small RNA consisting of 18-22 nucleotides. Accumulated evidence indicates that IncRNAs can sponge 
miRNAs, thereby lead to the regulation of various cellular processes, such as cell proliferation, migration, invasion, differentiation and apoptosis $(7,8)$.

KCNQ10T1 is responsible for the suppression in a cluster of genes in cis, and has been documented to participate the pathogenesis of multiple cancers $(9,10)$. A recent study by Feng, et al. provides evidence for KCNQ10T1 as an oncogene in BCa by promoting tumor cell proliferation, migration and invasion, through sponging miR-145 (11). Our study predicted a binding site of KCNQ10T1 at the seed sequence of miR-935. The role of miR-935 has been investigated in some human malignancies, including renal cell carcinoma (12), colorectal carcinoma (13) and non-small cell lung cancer (14). In an in silico study by Zhu et al., the BCa cells with chemotherapy resistance had significantly lower miR-935 expression than normal cells (15), which may imply that miR-935 might be involved in the progression of BCa. Nevertheless, it is still known little about the role of miR-935 in BCa.

Considering the potential relationship between KCNQ10T1 and miR-935, this study was performed with the purpose to investigate the functional role miR-935 in the molecular mechanism underlying the regulatory effect of KCNQ10T1 on BCa progression. In addition, the expression of the KCNQ10T1/miR935 axis was further evaluated, as well as its performance in diagnosing BCa patients. The results of this study may provide a novel insight in the pathogenesis of $\mathrm{BCa}$, and the KCNQ10T1/miR-935 axis may contribute to the development of targeted BCa therapy.

\section{Materials And Methods}

\section{Cell culture and transfection}

BCa cell lines MCF-7, MDA-MB-231, BT474 and T47D, as well as a normal mammary epithelial cell line MCF-10A were obtained from Chinese Academy of Science (Shanghai, China). The four BCa cell lines were cultured in Dulbecco's modified Eagle's medium (DMEM; Gibco, NY, USA) supplemented with 10\% fetal bovine serum (FBS; Gibco). The MCF-10A were cultured in PRMI-1640 (Gibco) supplemented with $10 \%$ FBS. All cells were maintained in an incubator with $5 \% \mathrm{CO}_{2}$ at $37^{\circ} \mathrm{C}$.

The overexpression vector pcDNA3.1-KCNQ10T1 was synthesized by Genecopoeia (Guangzhou, China), and the miR-935 mimic and mimic negative control (NC) were obtained from GenePharma (Shanghai, China). The above vectors were transfected into BCa cells using Lipofectamine 3000 (Invitrogen; Thermo Fisher Scientific, Inc.) following the manufacturer's instruction. The subsequent cell experiments were carried out at $48 \mathrm{~h}$ after cell transfection.

\section{Patients and tissue collection}

A total of 124 patients, who were pathologically diagnosed with BCa between October 2015 and April 2018 at Zibo Central Hospital, were included in the present study. None of the patients had received any antitumor therapy. During the same time period, 62 healthy volunteers were recruited to serve as healthy controls. Venous blood was collected from the participants, and serum samples were extracted by 
centrifugation and stored at $-80^{\circ} \mathrm{C}$ for further use. The written informed consent was obtained from each participate, and this study was approved by the Ethics Committee of Zibo Central Hospital.

\section{RNA extraction and quantitative real-time PCR (qRT-PCR)}

Total RNA was isolated from cells or serum using TRIzol reagent (Invitrogen, USA), then was reverse transcribed into cDNA with a reverse transcription kit PrimeScript RT Reagent Kit (TaKaRa, Dalian, China). qRT-PCR was carried out to measure the expression of KCNQ10T1 and miR-935 using the SYBR Green PCR Master Mix (TaKaRa, Dalian, China) on an ABI Prism 7500 Fast Real-Time PCR system (Applied Biosystems, Foster City, CA, USA). For KCNQ10T1 expression, GAPDH served as an internal control, and U6 was used as the endogenous control of miR-935. The final expression values were calculated using the $2^{-\Delta \Delta C t}$ method.

\section{Luciferase reporter assay}

The wild-type (WT) KCNQ10T1 with potential miR-935 binding site or the mutant-type (MUT) KCNQ10T1 was cloned into pMIR-REPORT plasmids (Promega, WI, USA). MCF-7 cells were seeded into 24-well plates and co-transfected with luciferase reporter plasmids and miR-935 mimic, miR-935 inhibitor or the negative controls using Lipofectamine 3000 (Invitrogen). At $48 \mathrm{~h}$ after transfection, the relative luciferase activity was measured using a dual luciferase reporter assay system (Promega) and normalized to the Renilla luciferase activity.

\section{Cell proliferation assay}

The proliferation of MCF-7 and MDA-MB-231 BCa cells was analyzed using a Cell Counting Kit-8 (CCK-8; Dojindo, Kumamoto, Japan). The cells with a density of $4 \times 10^{3}$ cells/well were seeded into 96 -well plates and incubated at $37^{\circ} \mathrm{C}$ for $72 \mathrm{~h}$. A volume of $10 \mu \mathrm{l}$ CCK-8 solution was added into the cells at $0,24,48$ and $72 \mathrm{~h}$, followed by a further $2 \mathrm{~h}$ of incubation. The absorbance at $450 \mathrm{~nm}$ was measured in each well using a microplate reader.

\section{Cell migration and invasion analysis}

The migratory and invasive capacities of MCF-7 and MDA-MB-231 cells were analyzed using Transwell assay ( $8 \mu \mathrm{m}$ pore size; Corning, NY, USA). The chambers used for invasion assay needed to be precoated with Matrigel (BD Biosciences, San Jose, CA, USA). After $48 \mathrm{~h}$ of transfection, the cells in serum-free medium were seeded in the upper chambers. The lower chambers included culture medium with $10 \%$ FBS as chemoattractant. After $24 \mathrm{~h}$ of incubation, the migratory or invasive cells were stained with $1 \%$ crystal violet and counted from five random fields under a light microscope (Olympus, Toyko, Japan).

\section{Statistical analysis}

All experiments were performed independently at least three times each time. All quantified data were presented as mean \pm SD, and the SPSS statistical software (version 20.0; IBM SPSS, USA) and GraphPad Prism software (version 7.0; GraphPad, USA) were applied for statistical analyses. Differences between groups were assessed using Student's t-test or one-way ANOVA followed by Tukey's post-hoc test. The 
correlation analysis was performed using Pearson correlation coefficient. A receiver operating characteristic (ROC) curve was plotted to evaluate the diagnostic value of the KCNQ10T1/miR-935 axis in BCa patients. A $P<0.05$ were considered statistically significant.

\section{Results}

\section{KCNQ10T1 directly binds to miR-935 and inhibits its expression}

A binding site of miR-935 was found at the sequence of KCNQ10T1 using the DIANA-LncBase v.2 (http://carolina.imis.athena-innovation.gr/diana_tools/) (16) (Fig. 1A). A subsequent luciferase reporter assay was used to confirm the interaction between KCNQ10T1 and miR-935. As shown in Fig. 1B, the relative luciferase activity was significantly inhibited by the overexpression of miR-935 in WT group $(P<$ 0.05), but no change was observed in the luciferase activity in MUT group. According to qRT-PCR, the expression of KCNQ10T1 in BCa cells was markedly higher in BCa cell lines than that in the normal cells (all $P<0.01$ ), while the expression of miR-935 was obviously decreased in $\mathrm{BCa}$ cells when compared to the controls (all $P<0.01$, Fig. 1C). Furthermore, KCNQ10T1 expression in BCa cells was upregulated by pcDNA3.1-KCNQ10T1 ( $P<0.001$, Fig. 1D), which leaded to a significant inhibitory effect on the expression of miR-935 $(P<0.01$, Fig. 1E). All these findings indicated that KCNQ10T1 could directly inhibit miR-935 in BCa cells.

\section{Effect of miR-935 on BCa cell proliferation, migration and invasion}

By cell transfection, the expression of miR-935 in MCF-7 and MDA-MB-231 BCa cells was successfully upregulated by the miR-935 mimic $(P<0.001$, Fig. $2 A)$. The absorbance results obtained from CCK-8 assay revealed that the overexpression of miR-935 led to an inhibition in cell proliferation in both two $\mathrm{BCa}$ cell lines (both $P<0.05$, Fig. 2B and $2 \mathrm{C}$ ). In addition, the Transwell assay results showed that the migration and invasion of $\mathrm{BCa}$ cells were also suppressed by the upregulation of miR-935 (all $P<0.01$, Fig. 2D and 2E).

\section{miR-935 mediates the regulatory function of KCNQ10T1 in BCa progression}

In MCF-7 and MDA-MB-231 cells, the expression miR-935 was reduced by the overexpression of KCNQ10T1, but this inhibition was abolished by the miR-935 mimic (all $P<0.01$, Fig. 3A). For cell proliferation, the overexpression of KCNQ10T1 in BCa cells resulted in the enhanced cell proliferation, while this effect was reversed by miR-935 upregulation (all $P<0.05$, Fig. 3B and 3C). Besides, the increased abilities of cell migration and invasion induced by pCDNA3.1-KCNQ10T1 were significantly decreased in the cells with overexpression of miR-935 (all $P<0.01$, Fig. 3D and 3E). 


\section{Serum expression of KCNQ10T1 and miR-935 in BCa patients}

The expression of KCNQ10T1 and miR-935 in serum samples collected from BCa patients was examined using qRT-PCR. As shown in Fig. 4A and 4B, we found that the serum KCNQ10T1 expression was significantly elevated, but the serum miR-935 was markedly reduced in $\mathrm{BCa}$ patients compared with healthy controls (both $P<0.001$ ). Additionally, a negative correlation was observed between the serum levels of KCNQ10T1 and miR-935 in patients with BCa $(r=-0.867, P<0.001$, Fig. 4C).

\section{Diagnostic performance of the KCNQ10T1/miR-935 axis in patients with $\mathrm{BCa}$}

Serum non-coding RNAs, especially miRNAs, have been considered a group of good diagnostic tools in human malignancies (17). The ROC curves based on serum KCNQ10T1 and miR-935 were constructed to evaluate their significance in BCa diagnosis. As shown in Fig. 5A, the aera under the curve (AUC) in a ROC based serum KCNQ10T1 levels was 0.900 with a sensitivity and a specificity of $85.5 \%$ and $82.3 \%$, respectively, under a cutoff value of 1.522. The ROC curve based on serum miR-935 expression showed an AUC of 0.919 , and the sensitivity and specificity were $93.5 \%$ and $85.5 \%$, respectively at a cutoff value of 0.834 (Fig. 5B). Furthermore, the combined diagnostic value of KCNQ10T1 and miR-935 performed a better diagnostic accuracy, which evidenced by the higher AUC (0.911) and improved sensitivity (95.4\%) and specificity (91.2\%) (Fig. 5C).

\section{Discussion}

Breast cancer is a malignancy that threatens women's health for a long time. Although there are many advanced therapeutic strategies till now, the clinical outcomes of BCa are still not ideal (18). Recent study showed that some molecules that involved in tumor progression could serve as biomarkers to improve the diagnosis and prognosis of patients with cancer, which had promoted the therapeutic strategies (19, 20). Among these functional molecules, IncRNAs and miRNAs are considered a group of important component, owing to their significant clinical significance and biological function $(21,22)$. For example, the increased expression of serum IncRNA UFC1 has been demonstrated to serve as a diagnostic and prognostic biomarker in patients with pancreatic cancer (23). Another study by Shi et al. provides evidence for the elevated serum miR-629 as a biomarker in the diagnosis and prognosis of pancreatic cancer (24). In BCa, Li et al. have found that the expression of IncRNA NEAT1 was upregulated, but the miR-211 expression was downregulated in BCa tissues and cell lines, which may serve as potential therapeutic targets by regulating BCa progression (25). These previous studies implied that the dysregulation non-coding RNAs, such as IncRNAs and miRNAs, in human malignancies have potencies to improve disease diagnosis, prognosis and treatment. 
In the present study, we focused on the clinical and biological role of the IncRNA KCNQ10T1/miR-935 axis in BCa progression. The expression and biological function of KCNQ10T1 has been investigated in a study by Feng et al., which reported that KCNQ10T1 expression was elevated in BCa tissues and cells, and the overexpression of KCNQ10T1 could enhance BCa cell proliferation through the miR-145/CCNE2 pathway (11). In addition, the KCNQ10T1 has been reported to facilitate the chemoresistance of colon cancer cells (26) and regulate tumor cell proliferation and apoptosis of tongue cancer cells (27). Similarly, our analysis results also detected the upregulated KCNQ10T1 expression in BCa cells and serum samples, and found the promoting effect of KCNQ10T1 on BCa cell proliferation, migration and invasion. Regarding the molecular mechanisms underlying the roles of IncRNAs in human cancers, numbers miRNAs have been found to be regulated by IncRNAs and thereby mediate the biological function of IncRNAs (28). By the prediction with bioinformatics analysis, a binding site of miR-935 was found at the sequences of KCNQ10T1. miR-935 has been investigated in renal cell carcinoma (12), colorectal carcinoma (13) and non-small cell lung cancer (14), but little is known about its role in BCa.

According to the data of this study, the expression of miR-935 was decreased in BCa cell lines and serum samples, which was negatively correlated with KCNQ10T1. The luciferase activity results provided evidence for the direct interaction between KCNQ10T1 and miR-935. Furthermore, the overexpression of miR-935 in BCa cells led to decreased cell proliferation, migration and invasion, and the enhanced $\mathrm{BCa}$ cell biological processes induced by KCNQ10T1 were reversed by the overexpression of miR-935, indicating that miR-935 might be a tumor suppressor and mediate the regulatory effect of KCNQ10T1 on $\mathrm{BCa}$ cell proliferation, migration and invasion. A study Yu et al. has found that the elevated IncRNA CASC15 could accelerate BCa cell proliferation and invasion by sponging miR-153-3p (29). Another study by Lu et al. also reported the regulatory effect of IncRNA LINC00511 on BCa tumorigenesis through inhibiting miR-185-3p (30). The aforementioned studies indicate the important role and therapeutic potential of IncRNAs and miRNAs in the pathogenesis of BCa. Collectively, the KCNQ10T1/miR-935 axis analyzed in this study provided a novel insight into the pathologic mechanisms of $\mathrm{BCa}$, and the treatment of BCa might be improved by targeting this novel axis.

miRNAs have been widely investigated as a group of biomarkers in the diagnosis and prognosis in a variety of human cancers (31). The clinical significance of miR-935 has been reported in some malignancies. For instance, the aberrant expression of miR-935 in colorectal carcinoma tissues has been documented to serve a biomarker to predict the prognosis of cancer patients (13). Similarly, the decreased expression of miR-935 in non-small cell lung cancer also indicated a poor prognosis in cancer patients (14). In this study, the serum miR-935 was found to be downregulated in BCa patients. The further ROC analysis results showed that the serum miR-935 expression levels had relatively high diagnostic accuracy for the differentiation between BCa patients and healthy individuals. Additionally, a ROC curve based on serum KCNQ10T1 also indicated that KCNQ10T1 might also had diagnostic potency in BCa patients. Considering the direct interaction between KCNQ10T1 and miR-935, the combined diagnostic accuracy of the two molecules was evaluated, which revealed that the synthetic use of KCNQ10T1 and miR-935 had an improved diagnostic accuracy compared with any single indicator. Taken together, our data might provide novel diagnostic biomarkers for patients with BCa. 
Although our study provided evidence for the biological function of the KCNQ10T1/miR-935 axis in BCa progression, the downstream molecules or signaling that involving in the function remain unclear, which may be the limitation of this study. Wang et al. has demonstrated that miR-935 served as a tumor suppressive miRNA in non-small cell lung cancer by targeting E2F7 and through the AKT signaling pathway (14). In BCa progression, whether the previously reported target genes and signaling also involved in the mechanisms underlying the role of KCNQ10T1/miR-935 axis warrant further investigations.

In conclusion, the findings in this study showed that miR-935, as a downstream target of KCNQ10T1, is downregulated in BCa cell lines and serum samples. KCNQ10T1 may accelerate BCa cell proliferation, migration and invasion by sponging miR-935, indicating the therapeutic potency of the KCNQ10T1/miR935 axis in BCa treatment. In addition, the serum reduced miR-935 and elevated KCNQ10T1 levels may be candidate biomarker for $\mathrm{BCa}$ diagnosis. All the data of this study may provide novel insight into the pathogenesis of $\mathrm{BCa}$ and novel method to improve disease treatment.

\section{Abbreviations}

IncRNAs, long non-coding RNAs

miRNAs, microRNAs

$\mathrm{BCa}$, breast cancer

qRT-PCR, quantative real-time PCR

FBS, fetal bovine serum

$\mathrm{NC}$, negative control

WT, wild-type

MUT, mutant-type

CCK-8, cell counting kit-8

ROC, receiver operating characteristic

\section{Declarations}

\section{Ethics approval and consent to participate}

The written informed consent was obtained from each participate, and this study was approved by the Ethics Committee of Zibo Central Hospital. 


\section{Consent for publication}

The consent for publication was obtained.

\section{Availability of data and material}

All data analyzed in this study are included in the manuscript.

\section{Competing interests}

The authors declare that they have no competing interests.

\section{Funding}

Not applicable.

\section{Authors' contributions}

CZ and JT made substantial contributions to conception and design, clinical analysis and interpretation of data, and was the major contributor in writing the manuscript. CG and JL involved in cell experiments and data analysis.

\section{Acknowledgements}

Not applicable.

\section{References}

1. Woolston C. Breast cancer. Nature. 2015;527(7578):S101.

2. Ghoncheh M, Pournamdar Z, Salehiniya H. Incidence and Mortality and Epidemiology of Breast Cancer in the World. Asian Pac J Cancer Prev. 2016;17(S3):43-6.

3. Bergh J, Hall P, Ostman A, Toftgard R. Breast cancer biology and the future of tailored therapies. Introduction. J Intern Med. 2013;274(2):102-4.

4. Odle TG. Precision Medicine in Breast Cancer. Radiol Technol. 2017;88(4):401M-21M.

5. Mohamed WA, Schaalan MF, Ramadan B. The expression profiling of circulating miR-204, miR-182, and IncRNA H19 as novel potential biomarkers for the progression of peptic ulcer to gastric cancer. J Cell Biochem. 2019;120(8):13464-77.

6. Wang ZH, Wang JH, Wang KQ, Zhou Y, Wang J. LncRNA FEZF1-AS1 promoted chemoresistance, autophagy and epithelial-mesenchymal transition (EMT) through regulation of miR-25-3p/ITGB8 axis in prostate cancer. Eur Rev Med Pharmacol Sci. 2020;24(5):2281-93.

7. Wei CM, Zhao XF, Qiu HB, Ming Z, Liu K, Yan J. The long non-coding RNA PVT1/miR-145-5p/ITGB8 axis regulates cell proliferation, apoptosis, migration and invasion in non-small cell lung cancer cells. Neoplasma. 2020. 
8. Ma YL, Wang CY, Guan YJ, Gao FM. Long noncoding RNA ROR promotes proliferation and invasion of colorectal cancer by inhibiting tumor suppressor gene NF2 through interacting with miR-223-3p. Eur Rev Med Pharmacol Sci. 2020;24(5):2401-11.

9. Xie Y, Wang M, Shao Y, Deng X, Chen Y. Long Non-coding RNA KCNQ10T1 Contributes to Antiepileptic Drug Resistance Through the miR-138-5p/ABCB1 Axis in vitro. Front Neurosci. 2019;13:1358.

10. Feng L, Li H, Li F, Bei S, Zhang X. LncRNA KCNQ1OT1 regulates microRNA-9-LMX1A expression and inhibits gastric cancer cell progression. Aging (Albany NY). 2020;12(1):707-17.

11. Feng W, Wang C, Liang C, Yang H, Chen D, Yu X, et al. The Dysregulated Expression of KCNQ10T1 and Its Interaction with Downstream Factors miR-145/CCNE2 in Breast Cancer Cells. Cell Physiol Biochem. 2018;49(2):432-46.

12. Liu F, Chen Y, Chen B, Liu C, Xing J. MiR-935 Promotes Clear Cell Renal Cell Carcinoma Migration and Invasion by Targeting IREB2. Cancer Manag Res. 2019;11:10891-900.

13. Huang Y, Xiao W, Jiang X, Li H. MicroRNA-935 acts as a prognostic marker and promotes cell proliferation, migration, and invasion in colorectal cancer. Cancer Biomark. 2019;26(2):229-37.

14. Wang C, Li S, Xu J, Niu W, Li S. microRNA-935 is reduced in non-small cell lung cancer tissue, is linked to poor outcome, and acts on signal transduction mediator E2F7 and the AKT pathway. Br J Biomed Sci. 2019;76(1):17-23.

15. Zhu QN, Renaud H, Guo Y. Bioinformatics-based identification of miR-542-5p as a predictive biomarker in breast cancer therapy. Hereditas. 2018;155:17.

16. Paraskevopoulou MD, Vlachos IS, Karagkouni D, Georgakilas G, Kanellos I, Vergoulis T, et al. DIANALncBase v2: indexing microRNA targets on non-coding transcripts. Nucleic Acids Res. 2016;44(D1):D231-8.

17. Bertoli G, Cava C, Castiglioni I. MicroRNAs: New Biomarkers for Diagnosis, Prognosis, Therapy Prediction and Therapeutic Tools for Breast Cancer. Theranostics. 2015;5(10):1122-43.

18. Peart O. Metastatic Breast Cancer. Radiol Technol. 2017;88(5):519M-39M.

19. Asiaf A, Ahmad ST, Arjumand W, Zargar MA. MicroRNAs in Breast Cancer: Diagnostic and Therapeutic Potential. Methods Mol Biol. 2018;1699:23-43.

20. Godone RLN, Leitao GM, Araujo NB, Castelletti CHM, Lima-Filho JL, Martins DBG. Clinical and molecular aspects of breast cancer: Targets and therapies. Biomed Pharmacother. 2018;106:14-34.

21. Oztemur Islakoglu Y, Noyan S, Aydos A, Gur Dedeoglu B. Meta-microRNA Biomarker Signatures to Classify Breast Cancer Subtypes. OMICS. 2018;22(11):709-16.

22. Liu Y, Sharma S, Watabe K. Roles of IncRNA in breast cancer. Front Biosci (Schol Ed). 2015;7:94-108.

23. Liu P, Sun QQ, Liu TX, Lu K, Zhang N, Zhu Y, et al. Serum IncRNA-UFC1 as a potential biomarker for diagnosis and prognosis of pancreatic cancer. Int J Clin Exp Pathol. 2019;12(11):4125-9.

24. Shi W, Lu Y, Gong R, Sun JJ, Liu G. Serum miR-629 is a novel molecular marker for diagnosis and the prognosis of pancreatic cancer. Eur Rev Med Pharmacol Sci. 2018;22(16):5187-93. 
25. Li X, Wang S, Li Z, Long X, Guo Z, Zhang G, et al. The IncRNA NEAT1 facilitates cell growth and invasion via the miR-211/HMGA2 axis in breast cancer. Int J Biol Macromol. 2017;105(Pt 1):346-53.

26. Li Y, Li C, Li D, Yang L, Jin J, Zhang B. IncRNA KCNQ10T1 enhances the chemoresistance of oxaliplatin in colon cancer by targeting the miR-34a/ATG4B pathway. Onco Targets Ther. 2019;12:2649-60.

27. Zhang S, Ma H, Zhang D, Xie S, Wang W, Li Q, et al. LncRNA KCNQ10T1 regulates proliferation and cisplatin resistance in tongue cancer via miR-211-5p mediated Ezrin/Fak/Src signaling. Cell Death Dis. 2018;9(7):742.

28. Chan JJ, Tay Y. Noncoding RNA:RNA Regulatory Networks in Cancer. Int J Mol Sci. 2018;19(5).

29. Yu L, Xu Q, Yu W, Duan J, Dai G. LncRNA cancer susceptibility candidate 15 accelerates the breast cancer cells progression via miR-153-3p/KLF5 positive feedback loop. Biochem Biophys Res Commun. 2018;506(4):819-25.

30. Lu G, Li Y, Ma Y, Lu J, Chen Y, Jiang Q, et al. Long noncoding RNA LINC00511 contributes to breast cancer tumourigenesis and stemness by inducing the miR-185-3p/E2F1/Nanog axis. J Exp Clin Cancer Res. 2018;37(1):289.

31. Armand-Labit V, Pradines A. Circulating cell-free microRNAs as clinical cancer biomarkers. Biomol Concepts. 2017;8(2):61-81.

\section{Figures}



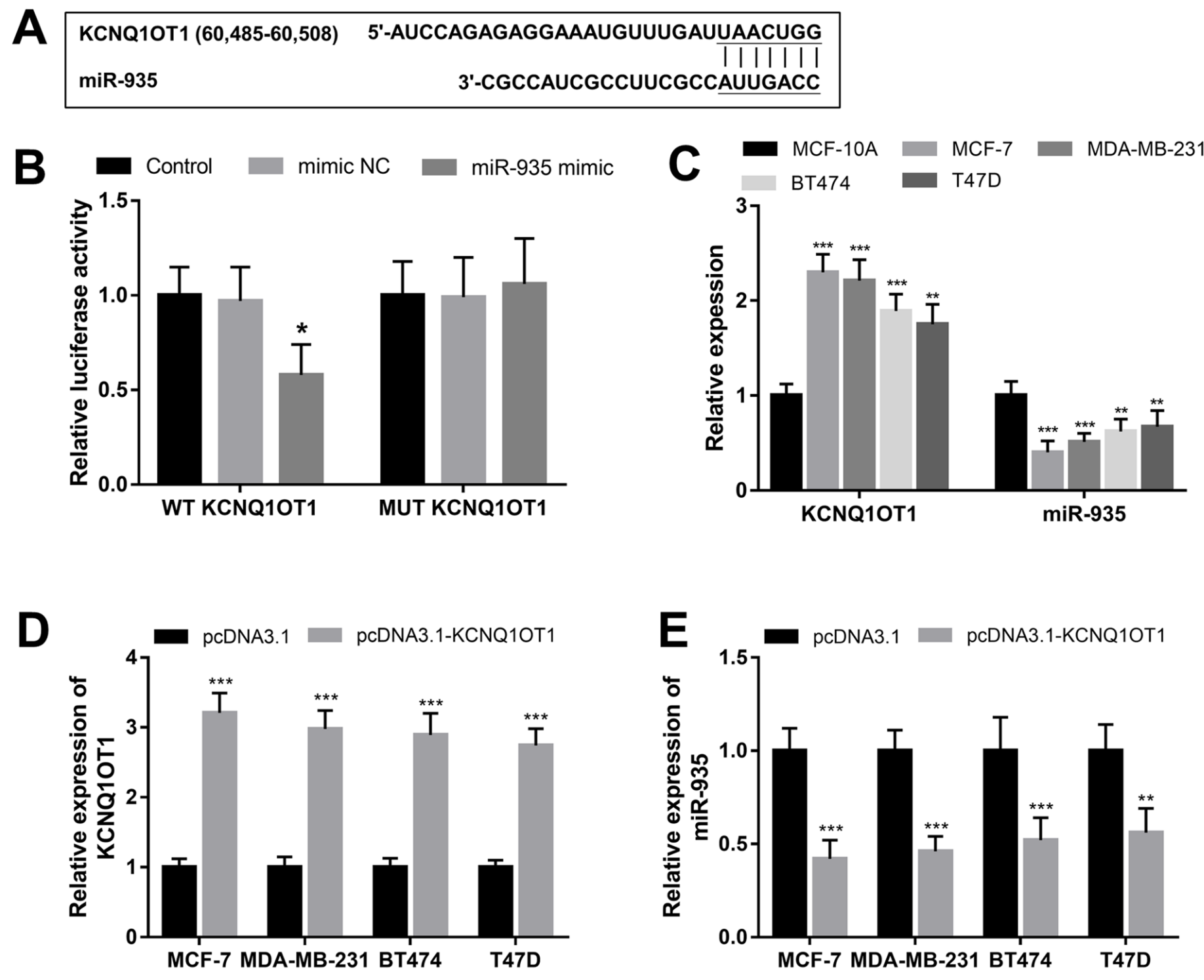

Figure 1

KCNQ10T1 directly regulates the expression of miR-935 in BCa cells. A. The binding site of miR-935 at the KCNQ10T1 sequence. B. Luciferase activity results to detect the interaction between KCNQ10T1 and miR-935 ( ${ }^{\mathrm{P}}<0.05$ vs. Control). C. Expression of KCNQ10T1 and miR-935 in BCa cell lines ( $* * P<0.01$, ***P $<0.001$ vs. MCF-10A). D. Expression of KCNQ10T1 was successfully upregulated by the pcDNA3.1KCNQ10T1 in BCa cell lines ( ${ }^{* * P}<0.001$ vs. pcDNA3.1). E. Expression of miR-935 was inhibited by the overexpression of KCNQ10T1 in the four BCa cell lines (***P $<0.001$ vs. pcDNA3.1). 

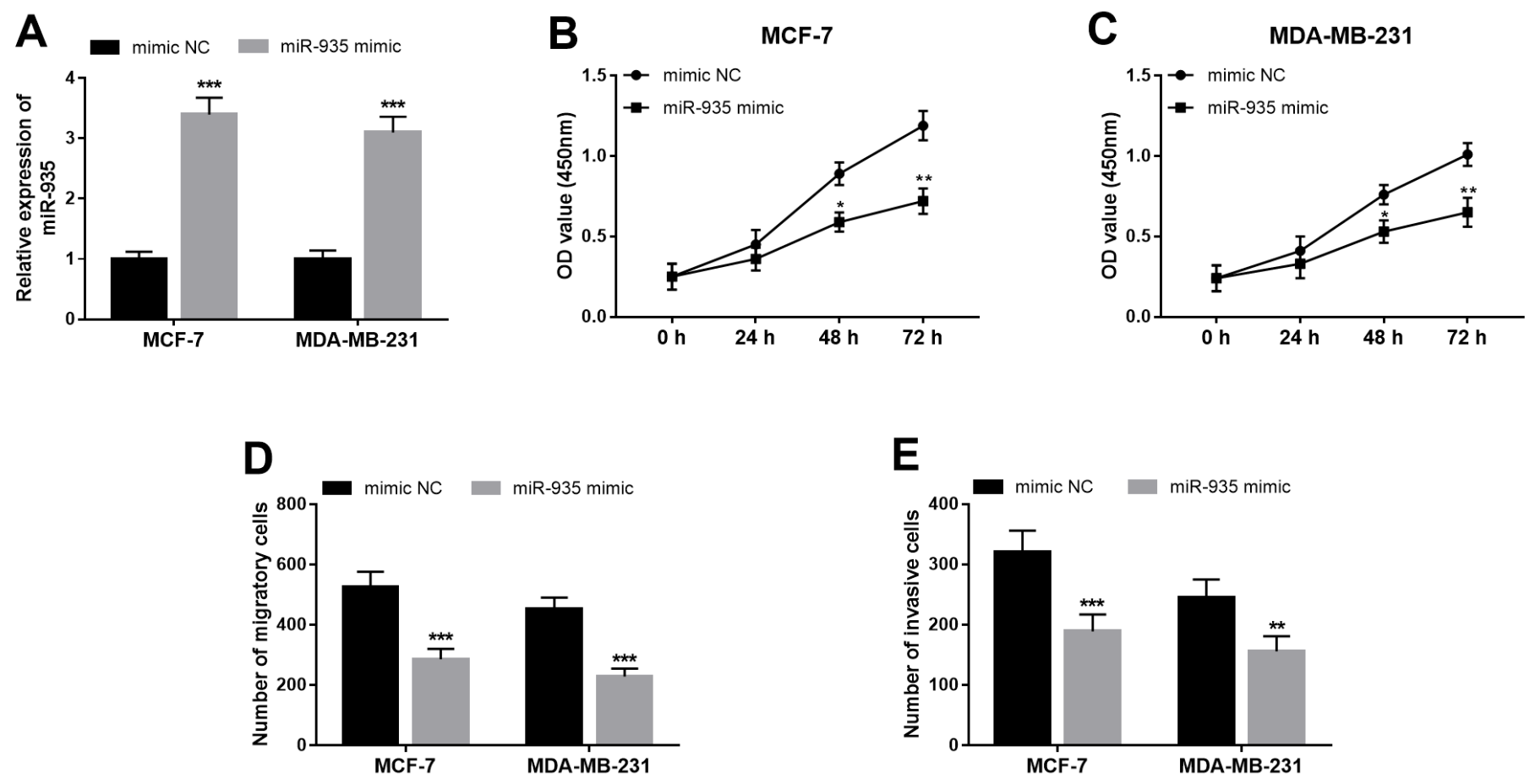

Figure 2

Effect of miR-935 on cell proliferation, migration and invasion of MCF-7 and MDA-MB-231 BCa cell lines. A. Expression of miR-935 was successfully upregulated by the miR-935 mimic ( ${ }^{\star *} \mathrm{P} P<0.001$ vs. mimic NC). B and C. BCa cell proliferation was inhibited by the overexpression of miR-935 in both the MCF-7 (B) and MDA-MB-231 (C) cell lines ( ${ }^{*} P<0.05,{ }^{*} \mathrm{P}<0.01 \mathrm{vs}$. mimic NC). D and $E$. The upregulation of miR-935 in $\mathrm{BCa}$ cells led to the suppressed cell migration $(D)$ and invasion $(E)(* * P<0.01, * \star * P<0.001$ vs. mimic $\mathrm{NC})$. 


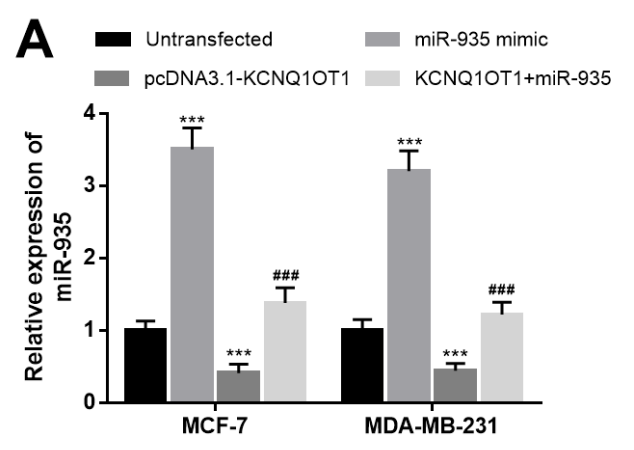

B

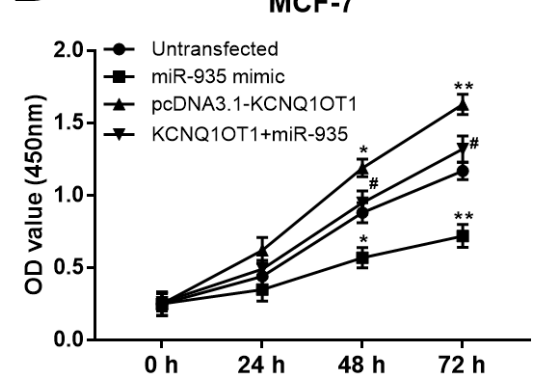

C

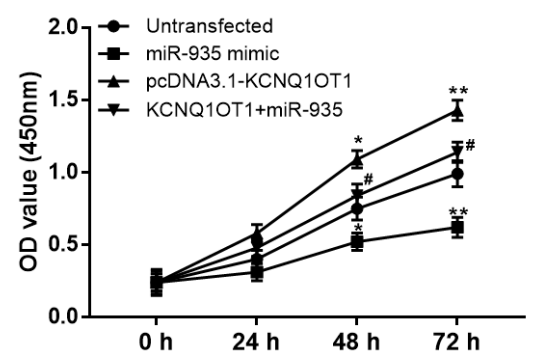

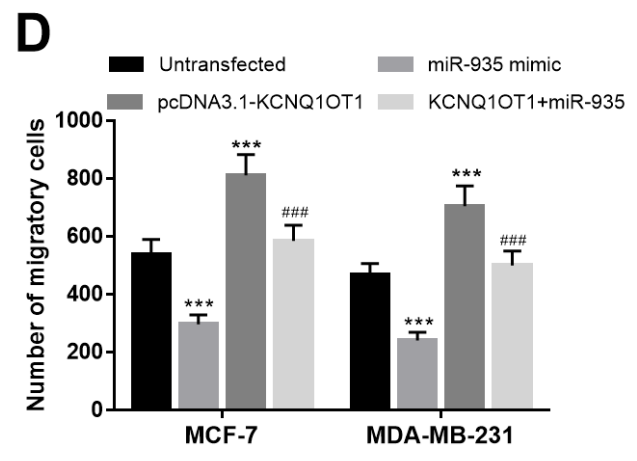

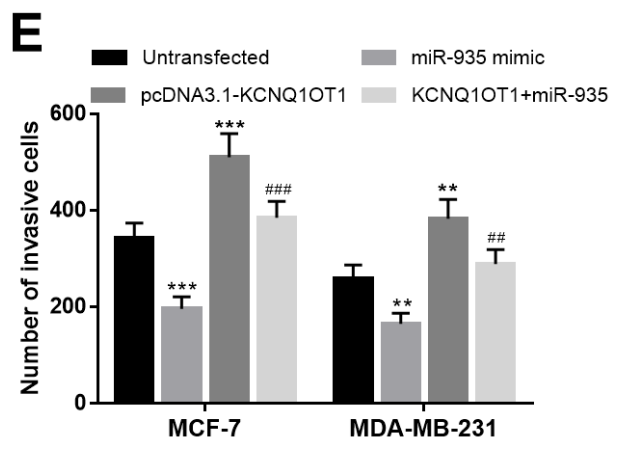

Figure 3

miR-935 mediates the biological role of KCNQ10T1 in the progression of BCa. A. Expression of miR-935 was inhibited by the overexpression of KCNQ10T1, but this inhibition was reversed by the miR-935 mimic. B and C. The overexpression of KCNQ10T1 (B) in both MCF-7 (C) and MDA-MB-231 cells led to enhanced cell proliferation, while this effect was abolished by the upregulation of miR-935. D and $\mathrm{E}$. The BCa cell migration (D) and invasion (E) abilities were promoted by the overexpression of KCNQ10T1, but this promoting effect was suppressed by the elevated miR-935. ${ }^{\star} \mathrm{P}<0.05$, ${ }^{\star *} \mathrm{P}<0.01$, ${ }^{\star \star *} \mathrm{P}<0.001$ vs. Untransfected; \#P $<0.05, \# \# P<0.01$, \#\#\# < 0.001 vs. pcDNA3.1-KCNQ10T1.
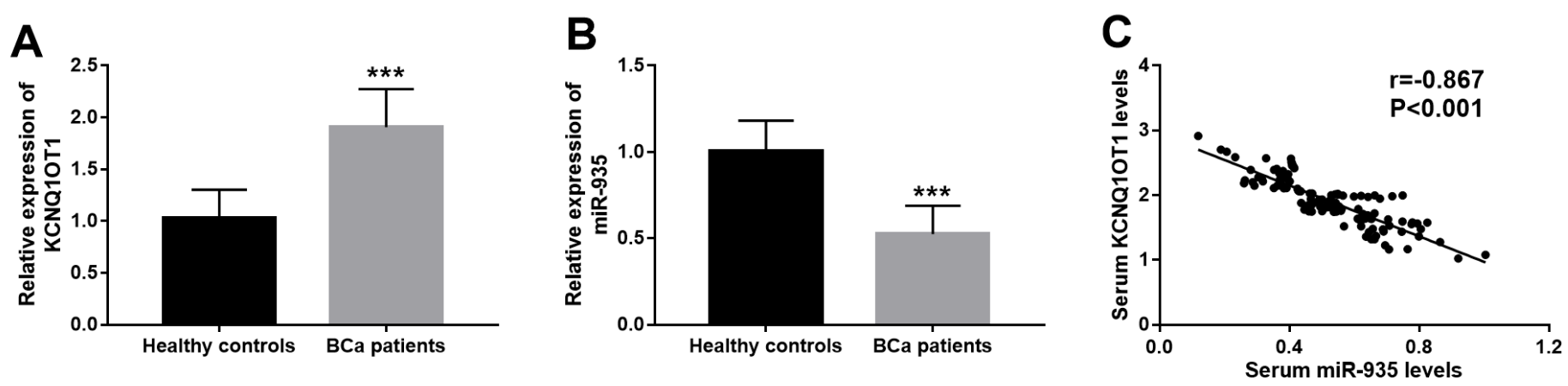

Figure 4

Serum KCNQ10T1 and miR-935 expression in patients with BCa. A. Serum expression of KCNQ10T1 was higher in BCa patients than that in healthy controls ( $* \star * P<0.001$ vs. Healthy control). B. Serum miR-935 expression was downregulated in BCa patients compared with healthy controls ( $* \star * P<0.001$ vs. Healthy 
control). C. Serum levels of KCNQ10T1 was negatively correlated with miR-935 in BCa patients ( $r=$ $-0.867, \mathrm{P}<0.001)$.
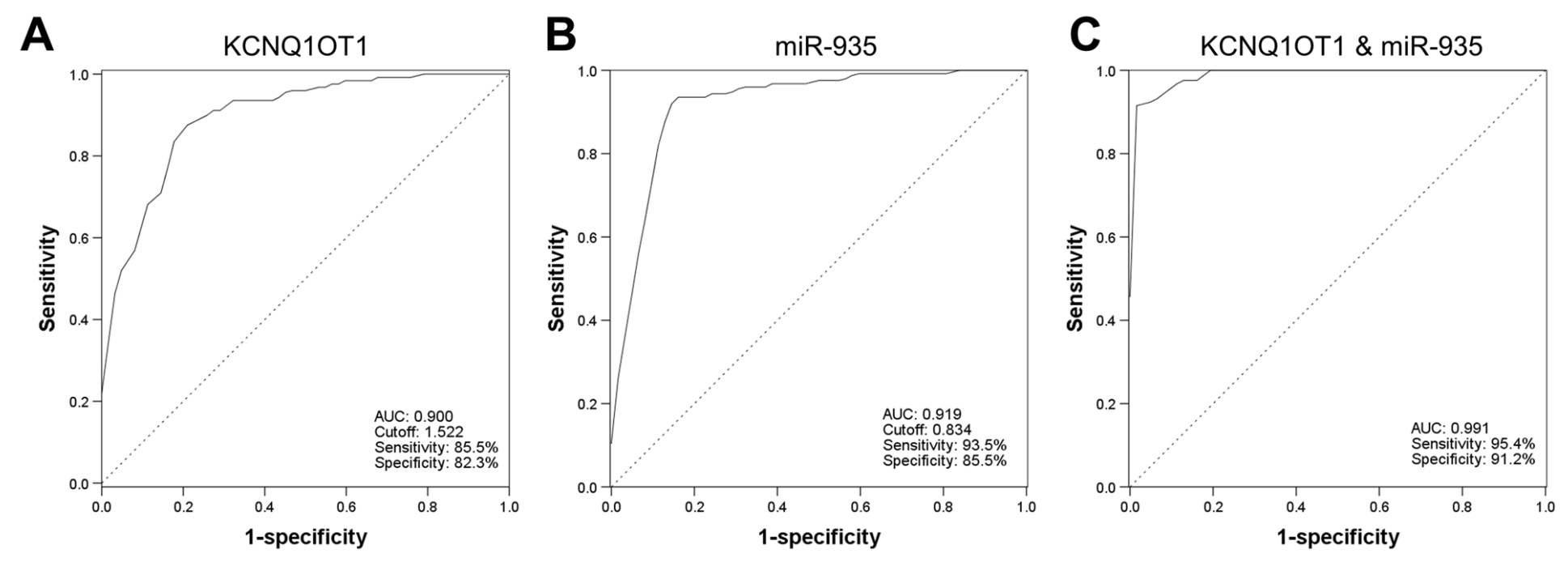

\section{Figure 5}

ROC curves based on serum KCNQ10T1 and miR-935 for BCa patients. A. A ROC curve constructed according to the serum levels of KCNQ10T1 in BCa patients. B. A ROC curves based on serum expression of miR-935 for BCa patients. C. A ROC curves based on the combined values of KCNQ10T1 and miR-935 in BCa patients. AUC, area under the curve. 